\title{
Measurement of Pneumococcal Polysaccharide Antibodies
}

\author{
Ricardo U. Sorensen • Lily E. Leiva
}

Received: 2 October 2013 / Accepted: 2 December 2013 / Published online: 17 December 2013

(C) Springer Science+Business Media New York 2013

Janssen et al in this journal published their paper "Measurement of pneumococcal polysaccharide vaccine responses for immunodeficiency diagnostics: combined IgG responses compared to serotype specific IgG responses". It is important to note that the message of this study is the observation that measuring antibodies against a mixture of all 23 pneumococcal serotype polysaccharides by ELISA does not reliably identify patients with a deficient response to pneumococcal polysaccharides. This holds true for patients with CVID, Specific Antibody Deficiency (SAD) or any other immunodeficiency affecting antibody production. Therefore, this study confirms a finding that is well established and beyond question; the only way to properly assess the ability of producing IgG anti-pneumococcal antibodies is to measure antibodies to each serotype individually. This is particularly important in the diagnosis of SAD since this deficiency is defined as an abnormal response to pure pneumococcal polysacchrides, as in the 23 -valent poneumococcal vaccine [1]. Now that most young patients are already vaccinated with a pneumococcal conjugate vaccine and may have developed antibodies to serotypes present in these vaccines, the global ELISA test would not be able to identify the lack of response to serotypes that are only in the 23-valent polysaccharide and not in the conjugate vaccines.

The study by Janssen et al does lend further clarification about the value of the Luminex method for the diagnosis of SAD. The Luminex method is now used by most commercial laboratories in the USA because it offers significant logistic advantages over the well-standardized but more cumbersome WHO ELISA method. Luminex allows the simultaneous

R. U. Sorensen $(\bowtie) \cdot$ L. E. Leiva

Department of Pediatrics, Louisiana State University Health Science

Center and Children's Hospital, 200 Henry Clay Ave., New Orleans,

LA, USA

e-mail: rsoren@1suhsc.edu quantitation of multiple serotype-specific antibodies. Both the third generation WHO ELISA and Luminex based tests offered in the USA incorporate double absorption of samples with cell wall polysaccharide (CPS) and serotype 22F.

The WHO ELISA test results have been shown to correlate closely with opsonophagocytosis (OPA) measurements [2, 3]. Although the Luminex method has been approved for the measurement of specific anti-pneumococcal serotype IgG antibodies, the correlation of this test with the standard ELISA test is poor and its use as $\mathrm{s}$ a diagnostic tool for SAD still needs further study. Recent studies designed to validate the Luminex assay for anti-pneumococcal IgG antibodies have shown different degrees of variability when compared to the ELISA method [4]. Results obtained in a recent comprehensive comparison of the multiplex Lumine ${ }^{\circledR}$ XMAP $^{\circledR}$ Pneumo 14 assay with the gold standard WHO ELISA in sera samples from children and adults taken pre- and post- $\operatorname{Prevnar}^{\circledR}$ and Pneumovax ${ }^{\circledR}$ immunization revealed that Luminex based results for individual serotypes are often different from results obtained by the ELISA test [5]. These authors concluded that when applying current AAAAI guidelines for the diagnosis of SAD [1], the Luminex xMAP Pneumo 14 assay does not allow reliable evaluation of antibody responses to polysaccharide antigens for the assessment of humoral immune competence. However, these authors also discussed that the xMAP system could be enhanced by using alternative interpretation criteria to determine an adequate response to polysaccharide antigens using multiplex bead assays.

Other studies claim that although interlaboratory analytical variability between assays exists, this variability has a relatively minor impact on classifying patients as "protected" or "nonprotected" when using established diagnostic algorithms $[6,7]$. Borgers et al. [6] further suggests that if a post- immunization titer is above the 5 th percentile for post-immunization IgG titers determined in a healthy population. A normal response to greater than $50 \%$ of serotypes tested, could be 
deemed an adequate response. These authors propose that this approach correctly identified antibody deficient subjects. These criteria await confirmatory testing by other authors and acceptance by expert committees after careful analysis of the evidence presented.

The variable results obtained with ELISA and Luminex methods, despite the use of standard reference sera to calibrate them, emphasize that strict definition based on percentages of responses considered to be adequate or not adequate as proposed by the expert working group on interpretation of diagnostic vaccination in primary immunodeficiency [1] cannot be reliably assessed until further validation of the variable results obtained by the ELISA and the Luminex methods is performed. We conclude that patient's management decisions have to be based primarily on the clinical condition of the patient rather than just on anti-pneumococcal antibody results.

\section{References}

1. Orange J, Ballow M, Stiehm E, Ballas Z, Chinen J, De La Morena M, et al. Use and interpretation of diagnostic vaccination in primary immunodeficiency: a working group report of the Basic and Clinical Immunology Interest Section of the American Academy of Allergy, Asthma \& Immunology. J Allergy Clin Immunol. 2012;130(3 Suppl): S1-S24.

2. Concepcion NF, Frasch C. Pneumococcal type $22 \mathrm{~F}$ absorption improves the specificity of a pneumococcal-polysaccharide enzyme linked immunosorbent assay. Clin Diagn Lab Immunol. 2001;8:26672.

3. Henckaerts I, Goldblatt D, Ashton L, Poolman J. Critical differences between pneumococcal polysaccharide enzyme-linked immunosorbent assays with and without $22 \mathrm{~F}$ inhibition at low antibody concentrations in pediatric sera. Clin Vaccine Immunol. 2006;13(3):356-60.

4. Pickering J, Martins T, Greer R, Schroder M, Astill M, Litwin C, et al. A multiplexed fluorescent microsphere immunoassay for antibodies to pneumococcal capsular polysaccharides. Am J Clin Pathol. 2002;117: 589-96.

5. Balloch A, Licciardi PV, Tang ML. Serotype-specific antipneumococcal $\mathrm{IgG}$ and immune competence: critical differences in interpretation criteria when different methods are used. J Clin Immunol. 2013;33(2):335-41.

6. Borgers H, Moens L, Picard C, Jeurissen A, Raes M, Sauer K, et al. Laboratory diagnosis of specific antibody deficiency to pneumococcal capsular polysaccharide antigens by multiplexed bead assay. Clin Immunol. 2010;134:198-205.

7. Zhang X, Simmerman K, Yen-Lieberman B, Daly T. Impact of analytical variability on clinical interpretation of multiplex pneumococcal serology assays. Clin Vaccine Immunol. 2013;20:957-61. 